九州大学学術情報リポジトリ

Kyushu University Institutional Repository

\title{
ON A FUNDAMENTAL BOUND OF BALANCED ARRAYS
}

\section{Kageyama, Sanpe i}

Department of Mathematics, Faculty of School Education, Hiroshima University

https://doi.org/10.5109/13354

出版情報: Bulletin of informatics and cybernetics. 21 (1/2), pp.37-39, 1984-03. Research Association of Statistical Sciences

バージョン :

権利関係 : 


\title{
ON A FUNDAMENTAL BOUND OF BALANCED ARRAYS*
}

By

\author{
Sanpei KAGEYAMA**
}

\begin{abstract}
Balanced arrays of strength $t$ in $N$ assemblies with $m$ constraints and $s$ symbols are useful in the construction of fractional factorial designs and to various combinatorial areas of design of experiments. To construct such arrays with the maximum possible number, $m$, of constraints is a very important problem both in the statistical design of experiments and combinatorial mathematics. In this note, balanced arrays satisfying a bound $m \leqq N$ are completely characterized.
\end{abstract}

\section{Introduction}

Let $A$ be an $m \times N$ matrix whose elements are $0,1, \cdots$, or $s-1$. Consider the $s^{t}$ $t$-vector, $X=\left(x_{1}, x_{2}, \cdots, x_{t}\right)^{\prime}$, which can be formed where $x_{i}=0,1, \cdots, s-1$ for $i=$ $1,2, \cdots, t$, and associate with each vector $X$ a positive integer $\lambda\left(x_{1}, x_{2}, \cdots, x_{t}\right)$ which is invariant under any permutations of $\left(x_{1}, x_{2}, \cdots, x_{t}\right)$. If, for every $t$-rowed submatrix of $A$, the $s^{t}$ distinct vectors $X$ occur as columns $\lambda\left(x_{1}, x_{2}, \cdots, x_{t}\right)$ times, then the matrix $A$ is called a balanced array of strength $t$ in $N$ assemblies with $m$ constraints, $s$ symbols and index parameters $\lambda\left(x_{1}, x_{2}, \cdots, x_{t}\right)$. For short, this is denoted by $B A(m, N, s, t)$.

Rafter and Seiden [1] noticed that $m \leqq N$ holds for all balanced arrays. It appears that this statement is not correct in general. The inequality $m \leqq N$ is the fundamental bound on the number of constraints, and can also be derived by considering the meaning of an $s^{m}$ factorial design. In this note, we shall characterize completely balanced arrays of validating the bound $m \leqq N$.

\section{Discussions}

Let $O_{a \times b}$ and $J_{a \times b}$ be $a \times b$ matrices whose elements are all zero and unity, respectively. Let $I_{a}$ be the identity matrix of order $a$. In this case, we can show the following theorem :

THEOREM. In a $B A(m, N, s, t)$ with $t \geqq 2$ except for any juxtaposition of $O_{m \times l_{1}}, J_{m \times l_{2}}$, $2 J_{m \times l_{3}}, \cdots$, or $(s-1) J_{m \times l_{s}}$ satisfying $N \geqq l_{i} \geqq 0$ and $\sum_{i=1}^{s} l_{i}=N$, an inequality $m \leqq N$ always holds.

* Prepared while the author was visiting the Indian Statistical Institute, Calcutta, India, during August 1982 to March 1983.

** Department of Mathematics, Faculty of School Education, Hiroshima University, Shinonome, Hiroshima 734, Japan. 
Proof. Let $A$ be a $B A(m, N, s, t)$ with $\lambda\left(x_{1}, x_{2}, \cdots, x_{t}\right)$ for $t \geqq 2$. Then it is well known that $A$ is also a $B A(m, N, s, 2)$ with appropriate index parameters $\lambda^{*}\left(x_{1}, x_{2}\right)$. In this case, it can be shown that

with

$$
\begin{aligned}
\left|A A^{\prime}\right| & =\left|\left(a_{1}-a_{2}\right) I_{m}+a_{2} J_{m \times m}\right| \\
& =\left(a_{1}-a_{2}\right)^{m-1}\left\{a_{1}+(m-1) a_{2}\right\}
\end{aligned}
$$

$$
\begin{aligned}
& a_{1}=\sum_{x_{2}=0}^{s-1} \sum_{x_{1}=1}^{s-1} x_{1}^{2} \lambda^{*}\left(x_{1}, x_{2}\right), \\
& a_{2}=\sum_{x_{2}=1}^{s-1} \sum_{x_{1}=1}^{s-1} x_{1} x_{2} \lambda^{*}\left(x_{1}, x_{2}\right)
\end{aligned}
$$

and $a_{1} \geqq a_{2} \geqq 0$. If $\left|A A^{\prime}\right| \neq 0$, then it follows that

$$
m=\operatorname{rank}\left(A A^{\prime}\right)=\operatorname{rank}(A) \leqq N,
$$

i.e., an inequality $m \leqq N$ holds. Thus, we now investigate the possibility of $\left|A A^{\prime}\right|=0$ by considering two cases. Note that if $a_{2}=0$, then $a_{1} \geqq 0$. In this case if $a_{1}>0$, then $\left|A A^{\prime}\right| \neq 0$, and if $a_{1}=0$, then the following case (I ) comes out.

Case (I). $a_{1}=0$, which then implies $a_{2}=0$. Then $\left|A A^{\prime}\right|=0$. It is obvious that $a_{1}=0$ iff there only exist $\lambda^{*}\left(0, x_{2}\right)$ for some $x_{2}(=0,1, \cdots$, or $s-1)$. Furthermore, since $\lambda *\left(0, x_{2}\right)=\lambda^{*}\left(x_{2}, 0\right)$ from the definition of balanced arrays, it holds that $\lambda *\left(0, x_{2}\right)=0$ for all $x_{2}=1,2, \cdots, s-1$. Hence, there is the only possibility of the positive value of $\lambda^{*}(0,0)$, that is, the original array is of form $O_{m \times N}$.

Case (II). $a_{1} \div 0, a_{2} \neq 0$ and $a_{1}-a_{2}=0$. In this case, since $\lambda^{*}\left(x_{1}, x_{2}\right)=\lambda^{*}\left(x_{2}, x_{1}\right)$, it follows that

$$
\begin{aligned}
a_{1}-a_{2} & =\sum_{x_{x_{2}=0}=0}^{s-1} \sum_{\sum_{1}=1}^{s-1}\left(x_{1}^{2}-x_{1} x_{2}\right) \lambda^{*}\left(x_{1}, x_{2}\right) \\
& =\sum_{x_{2}=0}^{s-1} \sum_{x_{1}>x_{2}=1}^{s-1} b_{x_{1} x_{2}} \lambda *\left(x_{1}, x_{2}\right)
\end{aligned}
$$

where $b_{x_{1} x_{2}}$ 's are positive constants depending on values of $x_{1}$ and $x_{2}$. The relation $\left(^{*}\right)$ implies that if $a_{1}-a_{2}=0$, then there only exist some $\lambda^{*}(x, x)$ for $x=0,1,2, \cdots, s-1$. Thus, the original array will be only of form

$$
\left[O_{m \times l_{1}}: J_{m \times l_{2}}: 2 J_{m \times l_{3}}: \cdots:(s-1) J_{m \times l_{s}}\right]
$$

for non-negative integers $l_{i}$ satisfying $\sum_{i=1}^{s} l_{i}=N$. Other cases about $a_{i}$ 's always yield that $\left|A A^{\prime}\right| \neq 0$. Thus, the proof is completed.

When $s=2$ (two-symbol), the theorem yields the following.

COROLLARY. In a $B A(m, N, 2, t)$ with $t \geqq 2$ except for a type $\left[O_{m \times l}: J_{m \times(v-l)}\right]$ satisfying $N \geqq l \geqq 0$, an inequality $m \leqq N$ always holds.

REMARK. When $l=0$ and $N$, the two-symbol original balanced array will be $J_{m \times N}$ and $O_{m \times N}$, respectively.

A type of some juxtaposition of $O_{m \times l_{1}}, J_{m \times l_{2}}, 2 J_{m \times l_{3}}, \cdots$, or $(s-1) J_{m \times l_{s}}$ is a trivial 
balanced array for integers $l_{i}$ satisfying $N \geqq l_{i} \geqq 0$ and $\sum_{i=1}^{s} l_{i}=N$. In this sense, it follows that, in a non-trivial balanced array, the number of assemblies is always bounded below by the number of constraints.

\section{Acknowledgement}

The author wishes to express his thanks to Dr. G. M. Saha, Indian Statistical Institute, Calcutta, for his suggestion of a problem of this note, and to Dr. M. Kuwada, Hiroshima University, for his useful comments.

\section{References}

[1] Rafter, J.A. and Seiden, E.: Contributions to the theory and construction of balanced arrays, Ann. Statist. 2 (1974), 1256-1273.

Communicated by Ch. Asano

Received April 16, 1983 